\title{
Equivalent Conductor Layer for Fast 3D Finite Element Simulations of Inductive Power Transfer Coils
}

\author{
Alberto Delgado, Student Member, IEEE, Guillermo Salinas, Student Member, IEEE, \\ Jesus A. Oliver, Member, IEEE, Jose A. Cobos, Fellow, IEEE, and Jorge Rodriguez-Moreno.
}

\begin{abstract}
In this paper, a method to replace conductors, in one or multiple layers, by an equivalent layer with homogeneous current distribution is proposed to allow complex 3D Finite Element simulations. This equivalent layer needs a coarser mesh, then the simulations are accelerated. The electromagnetic properties of this equivalent layer will be defined to match skin and proximity losses of actual round and litz-wire conductors. Analytical and empirical equations to obtain these parameters will be provided and the method is validated in several inductive power transfer coils with 3D geometries.
\end{abstract}

Index Terms-Anisotropic model, Finite Element Analysis, Finite Element modeling, Inductive Power Transfer, Magnetic components, Wireless Power Transfer.

\section{INTRODUCTION}

$\mathbf{S}$ INCE the inductive coils have 3D field distribution, if the well-known analytical equations from the literature for calculating the winding losses in magnetic components [1][10] are used to approximate the losses in inductive coils, large error may be obtained because these methods are based on 1D field distribution.

Finite Element Tools allow solving electromagnetic 3D field distributions but require high computational cost, especially when the amount of conductors is high, or the diameter of the conductors is very small (even if both circumstances take place, for instance, Litz-wire conductors), because the mesh required to perform the simulation is excessively fine.

To overcome these mesh limitations, some researchers such as [11]-[14] perform a 3D simulation where the conductor is an ideal layer to obtain the inductance and coupling factor. This ideal layer occupies the same space as the $\mathrm{N}$ turns of the inductive coil (see Fig. 1) and the current excitation is homogeneous throughout the conductor. Therefore, AC resistance cannot be obtained because skin effect and proximity effect will not occur. To calculate the AC losses, they need to use analytical equations and Finite Element simulations. For instance, to calculate the proximity losses, they perform various detailed 2D simulations to obtain the magnetic field, then they calculate the losses of inductive coils using analytical equations.

A. Delgado, G. Salinas, J.A. Oliver and J.A. Cobos are with the Centro de Electrónica Industrial, Universidad Politécnica de Madrid, Madrid, 28602 SPAIN e-mail: a.delgado@upm.es, guillermo.salinas@upm.es, jesusangel.oliver@upm.es and ja.cobos@upm.es

J. Rodriguez-Moreno is with the Premo Group, Malaga, Spain e-mail: jorge.rodriguez@grupopremo.com

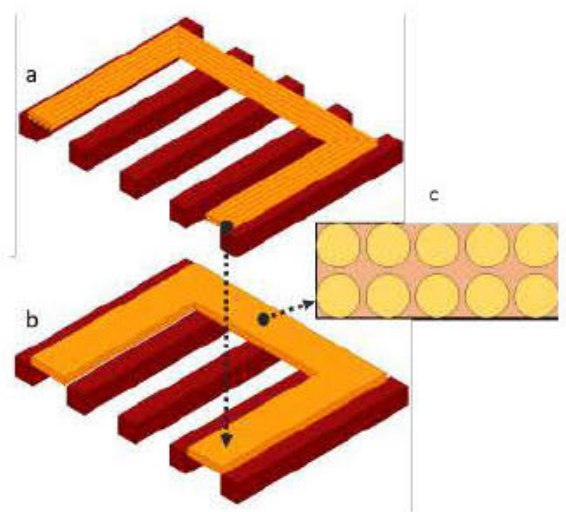

Figure 1: (a) 3D model of an inductive coil with a winding made of round conductors. (b) 3D Model of an inductive coil with a winding made of equivalent layer. (c) Cross section of the original layer.

However, the analytical equations to calculate the proximity effects have some limitations. For this reason, some researchers [15]-[19] perform a set of finite elements simulations to modify analytical equations from the literature. With these new expressions, it is possible to obtain good accuracy. However, to calculate the proximity losses, it is necessary to obtain the magnetic field which is affecting the winding using other analytical equation or from finite element simulations. These empirical equations are only accurate for 1D field distribution.

The Finite Element tool FEMM® [19] proposes an improved method with equivalent layers. This method can solve the problem using a continuum representation of wound coils by an equivalent layer. This method provides a real and imaginary conductivity in order to keep the losses and energy due to the skin effect; and a real and imaginary permeability in order to keep the losses and energy due to the proximity effect.

This approach has good accuracy when the winding is made with round wire and an hexagonally packed winding. Nevertheless, the approach is only valid when the distance among conductors are the same in all directions. Also, the imaginary part of the conductivity implies extra reactive power due to magnetic field energy stored in the winding. Then, after the simulation, this method needs the use of analytical equations to obtain the same actual energy in the winding. Something 
else we should notice is that FEMM® only simulates in $2 \mathrm{D}$ and limits the design process.

This work presents a method to allow direct 3D and 2D Finite Elements Analysis of inductive coils without postprocessing equations. Based on previous work [20], [21], we simplify the homogenization process: instead of performing the process in several steps, we describe a unique equation for each parameter. We also define a solution for cases when the winding is built with a single layer, which is not described in previous works.

The idea is to substitute the group of conductors (round or Litz-wire) utilized in the winding of a inductive coil by an equivalent layer with uniform current distribution which occupies the same area as the winding (see Fig. 1c). So that the meshing requirements are relaxed and fast 3D simulations are possible to obtain the inductance, coupling factor and losses of the inductive coil.

The conductivity of the equivalent layer is calculated to keep the losses due to the skin effect; the imaginary permeability will account for the proximity effect losses and the real permeability will keep the magnetic energy.

This paper is structured in four sections. In Section II, the analytical equations used in the homogenization process are described. In Section III, a brief qualitatively explanation of the homogenization process is presented. In Section IV, the homogenization process is quantitatively examined using the expressions described in Section II. In Section V, a set of finite element simulations using the homogenization process are performed and its results are compared with measurement.

\section{Magnetic AC Losses of the Conductors}

In order to obtain the magnetic and electrical properties of the equivalent layer proposed is this work, we need to know the losses of the conductors which are going to be substituted by the equivalent layer.

To this end, to calculate the losses in a conductor we have to consider two types of frequency dependent losses: skin $\left(P_{s e}\right)$ and proximity $\left(P_{p e}\right)$. In this work, both equations that define these effects are normalized by the DC resistance and expressed as:

$$
P_{s e}=F_{s} R_{D C} I^{2}
$$

And:

$$
P_{p e}=\frac{G_{p}}{\sigma} H^{2}
$$

Where $R_{D C}$ is the DC resistance of the conductors, $\sigma$ is the conductivity of the copper, $\mathrm{I}$ is the peak current that is exciting the conductor, $\mathrm{H}$ is the peak of the external magnetic field and $F_{s}$ and $G_{p}$ are factors that depend on the geometry, frequency and type of conductors.

In addition, if we have a layer with homogeneous current distribution we can use the integral form of the Poynting vector theorem [22] and replacing the permeability by $\mu=\mu^{\prime}-j \mu^{\prime \prime}$, we can obtain an expression to calculate the energy and the losses. The real part of the complex permeability $\mu^{\prime}$ is related to the energy, the conductivity $\sigma$ and the imaginary part of the complex permeability $\mu^{\prime \prime}$ are directly related to the power dissipation [23].
Thus, it is possible to equalize the averaged losses due to the skin effect in a conductor $\left\langle P_{\text {se }}\right\rangle$ and the term relates to the ohmic-losses of the layer as:

$$
\left\langle P_{s e}\right\rangle=\frac{1}{2} \int_{v} \sigma_{l} \vec{E}^{2} d v=\frac{1}{2} \int_{v} \frac{1}{\sigma_{l}} \vec{J}^{2} d v
$$

It is also possible to relate the averaged losses due to the proximity effect in a conductor $\left\langle P_{p e}\right\rangle$ that depends on the square of the magnetic field and the term given by the imaginary part of the complex permeability:

$$
\left\langle P_{p e}\right\rangle=\frac{1}{2} \int_{v} \mu_{l}^{\prime \prime} \omega \vec{H}^{2} d v
$$

Finally, it is possible to denote the averaged energy as:

$$
\langle E\rangle=\frac{1}{4} \int_{v} \mu_{l}^{\prime} \vec{H}^{2} d v
$$

Where $\mu_{l}$ and $\sigma_{l}$ are the complex permeability and the conductivity of the equivalent layer.

Therefore, to obtain the value of these parameters, the factors $F_{s}$ and $G_{p}$ from (1) and (2) must be known. In the following subsection we present the calculation of $F_{s}$ and $G_{p}$ for round and litz-wire conductors.

\section{A. Analytical calculation of round conductor}

The exact solution of round conductor with diameter $\mathrm{d}$ with a skin depth $\delta$ is developed in [1], [2]. The skin effect per unit length of a round conductor can be calculated with (1), where the exact expression for $F_{s}$ for a round conductor can be expressed as:

$$
\begin{aligned}
F_{s}(\gamma)=\frac{\gamma}{4 \sqrt{2}} & {\left[\frac{\operatorname{ber}_{0}(\gamma) \operatorname{bei}_{1}(\gamma)-\operatorname{ber}_{0}(\gamma) \operatorname{ber}_{1}(\gamma)}{\operatorname{ber}_{1}^{2}(\gamma)+\operatorname{bei}_{1}^{2}(\gamma)}\right.} \\
& \left.-\frac{\operatorname{bei}_{0}(\gamma) b e r_{1}(\gamma)+\operatorname{bei}_{0}(\gamma) b e i_{1}(\gamma)}{\operatorname{ber}_{1}^{2}(\gamma)+b e i_{1}^{2}(\gamma)}\right]
\end{aligned}
$$

Where $\gamma$ is $d / \sqrt{2} \delta$ and ber $_{i}$ and $b e i_{i}$ are the real and imaginary part of the Kelvin functions of order $i$.

Also, the proximity effect per length of a round conductor which is under the influence of an external magnetic field can be obtained using (2), where the exact expression for $G_{p}$ can be calculated as:

$$
\begin{aligned}
G_{p}(\gamma)=-\frac{2 \gamma \pi}{\sqrt{2}}\left[\frac{\operatorname{ber}_{2}(\gamma) \operatorname{ber}_{1}(\gamma)+\operatorname{ber}_{2}(\gamma) \operatorname{bei}_{1}(\gamma)}{\operatorname{ber}_{0}^{2}(\gamma)+\operatorname{bei}_{0}^{2}(\gamma)}\right. & \\
& \left.+\frac{\operatorname{bei}_{2}(\gamma) \operatorname{bei}_{1}(\gamma)-\operatorname{bei}_{2}(\gamma) \operatorname{ber}_{1}(\gamma)}{\operatorname{ber}_{0}^{2}(\gamma)+b e i_{0}^{2}(\gamma)}\right]
\end{aligned}
$$

The factor $F_{s}$ can be used for any conductor regardless of the ratio of skin depth to diameter of the conductor and the number of conductors. However, the accuracy of $G_{p}$ is low when $\delta \ll d$ and the conductors are close (affecting magnetic field distribution).

In the case of litz wire conductors, where the skin depth is much lower than the diameter, the accuracy of $F_{s}$ and $G_{p}$ is not compromised. However, to approximate the losses of a winding made of round conductors, the $G_{p}$ is not feasible. 


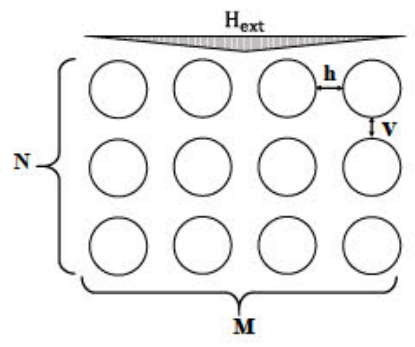

(a)

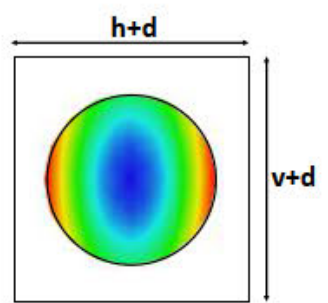

(b)
Figure 2: Finite Element Simulation configuration of Sullivan's Method: a) A group of conductors separated by horizontal distance $\mathrm{h}$ and vertical distance v. b) A cell of one conductor.

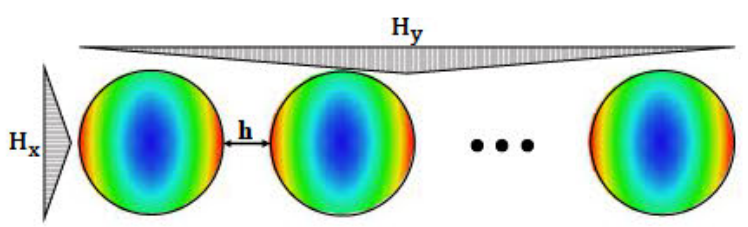

Figure 3: Finite Element Simulation configuration of OneLayer configuration.

\section{B. Finite Element Fitting of $G_{p}$}

To overcome the problems of the round conductor with the proximity effect described in the previous method, in [15] a modification of analytical equations is proposed. Based on empirical results from a Finite Element tool, it is modified a equation from literature to approximate the proximity effect of a winding configuration as can be shown in Fig. 2 at higher frequencies.

Using (2) to calculate the proximity losses, the empirical expression for $G_{p}$ proposed by [15] is the following:

$$
\begin{aligned}
G_{p}(X, v, h)= & G_{1}(X, v, h)(1-w(v, h)) \\
& +G_{2}(X, v, h) w(v, h)
\end{aligned}
$$

Where $X$ is $\gamma \sqrt{2}, v$ and $h$ are the distances between conductors in the vertical and horizontal axis (see Fig. 2) and

$$
\begin{aligned}
G_{1}(X, v, h)=\frac{3 \pi}{16} k(v, h)^{-3} X \\
{\left[\frac{\sinh (k(v, h) X)-\sin (k(v, h) X)}{\cosh (k(v, h) X)+\cos (k(v, h) X)}\right] } \\
G_{2}(X, v, h)=\frac{\pi}{32} \frac{X}{X^{-3}+b(v, h)^{3}}
\end{aligned}
$$

Where $k, b$ and $w$ depend on $v$ and $h$ and are given in [15].

This method can be applied to windings which are configured with several layers of conductors as in Fig. 2a. Nevertheless, in the case of single layer winding, it is $\mathrm{n}=1$ (or $\mathrm{m}=1$ ) as can be seen in Fig. 3, this equation cannot be applied. For this reason, in the following section, a new equation is proposed to allow the simulations of this type of windings configuration.

The modification is precise when the field is unidirectional or when the distances $h$ and $v$ are equal (see Fig.2); nevertheless, in this work we propose the use of the perpendicularity of the magnetic field to use this equation for bidirectional fields. Since the magnetic field can be decomposed in the sum of two 1D magnetic field, it is possible to obtain an equivalent layer with anisotropic complex permeability: one for the field in $\mathrm{x}$-direction and other for the field in y-direction.

\section{One-layer modification}

The modification of Dowell's equation does not consider when the winding is made of a single layer, it is $\mathrm{n}=1$ (or $\mathrm{m}=1$ ) in Fig. 2. In this work, we presented a new $G_{p}$ to calculate the proximity losses in a one-layer winding made of round conductors.

The one-layer setup considers a winding made of round conductors in a plane separated by a constant distance $h$. This one-layer is excited by a homogeneous magnetic field which can be applies in two directions (see Fig. 2).

The empirical expressions to calculate the proximity losses $G_{p_{i}}$ are described in Appendix A, and can be expressed as:

$$
\begin{aligned}
& G_{p_{x}}\left(\gamma, \frac{h}{d}, N_{\text {turns }}\right)= \\
& \quad x\left(\frac{h}{d}, N_{\text {turns }}\right) \frac{G_{1}(\gamma)}{f(\gamma)+1}+\frac{G_{2}(\gamma)}{f(\gamma)-1}
\end{aligned}
$$

And:

$$
\begin{aligned}
& G_{p_{y}}\left(\gamma, \frac{h}{d}, N_{\text {turns }}\right)= \\
& \quad y\left(\frac{h}{d}, N_{\text {turns }}\right) \frac{G_{1}(\gamma)}{f(\gamma)+1}+\frac{G_{2}(\gamma)}{f(\gamma)-1}
\end{aligned}
$$

\section{Parameters Calculation of the Equivalent LAYER}

The homogenization process starts by replacing of the conventional conductors, with their own current distribution and the surrounding air (see Fig. 4a, Fig. 5a and Fig. 6a) by a single equivalent layer which will have a homogeneous current distribution as can be seen in Fig. 4b, Fig. 5b and Fig. $6 \mathrm{~b}$. By doing so, the mesh is reduced substantively and the computational resources to perform finite element simulations are reduced.

Since this new equivalent layer has uniform current distribution, electromagnetic finite element simulation will provide conduction losses and magnetic field losses according to the calculated conductivity and the imaginary part of the complex permeability of the equivalent layer.

\section{A. Multiple layers of round conductor}

In order to obtain the homogeneous conductivity of the equivalent layer, the averaged losses due to the skin effect of the equivalent layer (3) and the skin-effect losses equation (1) in combination with (6) are equalized:

$$
\frac{1}{2} \int_{A_{l}} \frac{1}{\sigma_{l}} \vec{J}^{2} d A=N_{\text {turns }} F_{s}(\gamma) R_{D C} I^{2}
$$

Thus, the homogeneous conductivity of the equivalent layer can be expressed as:

$$
\sigma_{l}=\frac{1}{2} \frac{N_{\text {turns }}}{A_{l} F_{s}(\gamma) R_{D C}}
$$




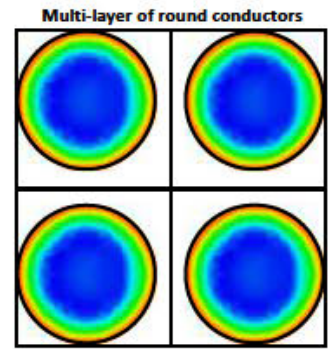

(a)

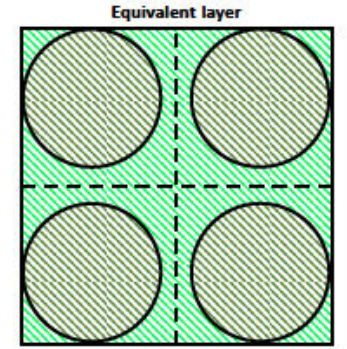

(b)
Figure 4: a) A representation of a multiple layers of round conductors under the frequency influence. b) The equivalent layer of multiple layers made of homogenized conductors.

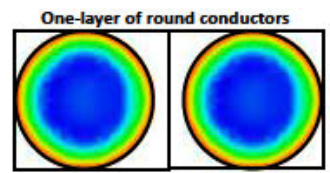

(a)

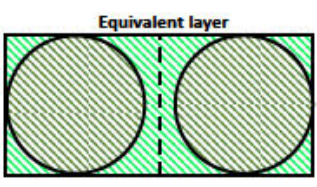

(b)
Figure 5: a) A representation of one-layer winding of round conductors under the frequency influence. b) The equivalent layer of a one-layer made of homogenized conductors.

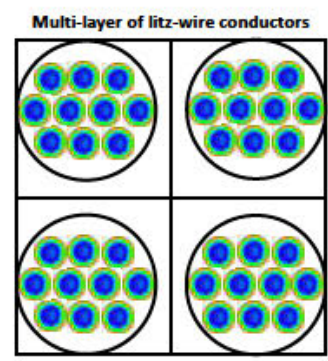

(a)

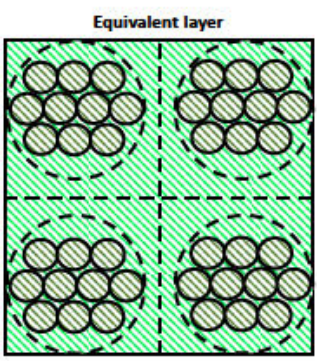

(b)
Figure 6: a) A representation of a multiple layers of Litz-wire conductors for a given frequency influence. b) The equivalent layer of a multiple layers made of homogenized conductors.

Where $R_{D C}$ is the DC resistance of one conductor per unit length and $A_{l}$ is the area of the equivalent layer.

To obtain the complex permeability of the equivalent layer for multiple layers of round conductor, we equate the averaged loses due to the magnetic field in $\mathrm{x}$ and $\mathrm{y}$ direction of the equivalent layer (4) and the proximity effect equation (2) in combination with (8):

$$
\frac{1}{2} \int_{A_{l}} \mu_{l x}^{\prime \prime} \mu_{0} \omega H_{x}^{2} d A=N_{t u r n s} \frac{G_{p}(X, h, v)}{\sigma} H_{x}^{2}
$$

$$
\frac{1}{2} \int_{A_{l}} \mu_{l y}^{\prime \prime} \mu_{0} \omega H_{y}^{2} A=N_{t u r n s} \frac{G_{p}(X, v, h)}{\sigma} H_{y}^{2}
$$

Simplifying both expressions, we can obtain the imaginary part of the complex permeability:

$$
\begin{array}{r}
\mu_{l x}^{\prime \prime}=\frac{2 N_{\text {turns }} G_{p}(X, h, v)}{\sigma \omega \mu_{0} A_{l}} \\
\mu_{l y}^{\prime \prime}=\frac{2 N_{\text {turns }} G_{p}(X, v, h)}{\sigma \omega \mu_{0} A_{l}}
\end{array}
$$

Where $N_{\text {turns }}$ is the number of turns in the winding and $A_{l}$ is the total area of the equivalent layer.

It can be noticed that the parameters $v$ and $h$ in (17) and (18) are swapped to take into account that the separation between conductors is different in $\mathrm{x}$ and $\mathrm{y}$ direction.

To obtain the real part of the complex permeability, it is possible to use the Kramers-Kronig relation using the imaginary part:

$$
\mu_{l_{i}}^{\prime}=\frac{2}{\pi} \int_{0}^{\infty} \frac{\mu_{l_{i}}^{\prime \prime}(x) x}{x^{2}-\omega^{2}} \partial x+\mu_{c t e}^{\prime}
$$

Where $\mu_{c t e}^{\prime}$ is calculated so that ensures the real permeability of the equivalent layer is 1 in low frequency and $i$ is (x,y)direction.

\section{B. One-layer of round conductor}

To obtain the homogeneous conductivity of each conductor, the expression (14) can be used.

For one-layer winding, the only difference is the calculation of $G_{p}$. In this case, (11) and (12) have to be used.

Then, the imaginary part of the complex permeability of the equivalent layer for both directions can be expressed as:

$$
\begin{gathered}
\mu_{l_{x}}^{\prime \prime}=\frac{2 G_{p_{x}}\left(N_{t u r n s}, \gamma, \frac{h}{d}\right)}{\sigma \omega \mu_{0} A_{l}} \\
\mu_{l_{y}}^{\prime \prime}=\frac{2 G_{p_{y}}\left(N_{t u r n s}, \gamma, \frac{h}{d}\right)}{\sigma \omega \mu_{0} A_{l}}
\end{gathered}
$$

Where $N_{\text {turns }}$ is the number of turns in the equivalent layer and $A_{l}$ is the total area of the equivalent layer.

The real part can be calculated using (42).

\section{Layers of Litz-wire conductors}

Equating averaged losses due to the skin-effect of an equivalent layer (3) and the skin-effect equation (1) in combination with (6) are equated:

$$
\sigma_{l}=\frac{N_{\text {turns }} N_{\text {strands }}}{2 A_{l} F_{s}(\gamma) R_{D C}}
$$

Where $\gamma$ is defined for one strand, $N_{\text {strands }}$ is the number of strands in the Litz-wire conductor, $N_{\text {turns }}$ the number of Litzwire conductors in the winding, $R_{D C}$ is the DC resistance of one strand per unit length and $A_{l}$ is the area of the equivalent layer that occupies one turn.

In addition, to obtain the homogeneous complex permeability, the equation (2) in combination with (7) is equated to the 
Table I: Equations of the Equivalent Layer Model

\begin{tabular}{|c|c|c|c|}
\hline Winding & Conductivity & Imaginary Permeability & Real Permeability \\
\hline Multi-Layer & $\sigma_{l}^{\prime}=\frac{1}{2} \frac{N_{t u r n s}}{A_{l} F_{s}(\gamma) R_{D C}}$ & $\begin{array}{c}\mu_{l x}^{\prime \prime}=\frac{2 N_{t u r n s} G_{p}(X, h, v)}{\sigma \omega \mu_{0} A_{l}} \\
\mu_{l y}^{\prime \prime}=\frac{2 N_{t u r n s} G_{p}(X, v, h)}{\sigma \omega \mu_{0} A_{l}} \\
\mu_{l i}^{\prime \prime}=\frac{2 N_{t u r n s} G_{p}\left(N_{t u r n s}, \gamma, \frac{h}{d}\right)}{\sigma \omega \mu_{0} A_{l}}\end{array}$ & $\begin{array}{c}\mu_{l x}^{\prime}=\frac{2}{\pi} \int_{0}^{\infty} \frac{\mu_{l x}^{\prime \prime}(x) x}{x^{2}-\omega^{2}} d x+\mu_{\text {cte }}^{\prime} \\
\mu_{l y}^{\prime}=\frac{2}{\pi} \int_{0}^{\infty} \frac{\mu_{l y}^{\prime \prime}(x) x}{x^{2}-\omega^{2}} d x+\mu_{\text {cte }}^{\prime} \\
\mu_{l x}^{\prime}\left(N_{t u r n s}, \frac{h}{d}, \gamma\right)=1-b\left(N_{t u r n s}, \frac{h}{d}\right) \tanh \left(\sqrt{\gamma-1.5} a\left(N_{\text {turns }}, \frac{h}{d}\right)\right) \\
\mu_{l y}^{\prime}=1\end{array}$ \\
\hline Litz-Wire & $\sigma_{l}^{\prime}=\frac{1}{2} \frac{N_{\text {turns }} N_{\text {strands }}}{A_{l} F_{s}(\gamma) R_{D C}}$ & $\mu_{l}^{\prime \prime}=\frac{2 N_{\text {strands }} N_{t u r n s} G_{p}(\gamma)}{\sigma \omega \mu_{0} A_{l}}$ & $\mu_{l}^{\prime}=\frac{2}{\pi} \int_{0}^{\infty} \frac{\mu_{l}^{\prime \prime}(x) x}{x^{2}-\omega^{2}} d x+\mu_{c t e}^{\prime}$ \\
\hline
\end{tabular}

Table II: Computation time in seconds for each simulation. E.L.=Equivalent Layer; $\mathrm{M}=$ Meshing, $\mathrm{S}=$ Solver.

\begin{tabular}{|c|c|c|c|c|c|c|c|c|}
\hline \multirow{2}{*}{ Case } & \multicolumn{2}{|c|}{ E.L.3D } & \multicolumn{2}{|c|}{ Conductor 3D } & \multicolumn{2}{|c|}{ E.L.2D } & \multicolumn{2}{|c|}{ Conductor 2D } \\
\hline & M. & S. & M. & S. & M. & S. & M. & S. \\
\hline A & $16 "$ & $2 "$ & \multirow{4}{*}{\multicolumn{2}{|c|}{ N/A }} & 5 " & $0.3^{\prime \prime}$ & $N / A$ & $N / A$ \\
\hline B & $45 "$ & 4" & & & & & & \\
\hline $\mathrm{C}$ & $65 "$ & $8 "$ & & & & & & \\
\hline D & $22 "$ & 3 " & & & 4" & $0.2^{\prime \prime}$ & $43 "$ & $12 "$ \\
\hline
\end{tabular}

averaged proximity effect losses of the equivalent layer (4) as:

$$
\mu_{l}^{\prime \prime}=\frac{2 N_{\text {turns }} N_{\text {strands }} G_{p}(\gamma)}{\sigma \omega \mu_{0} A_{l}}
$$

In this case, it is possible to use the equation $G_{p}$ of (7) because $\gamma$ is much smaller than 1 .

To obtain the real part of the complex permeability, it is possible to use the Kramers-Kronig relation using the imaginary part:

$$
\mu_{l}^{\prime}=\frac{2}{\pi} \int_{0}^{\infty} \frac{\mu_{l}^{\prime \prime}(x) x}{x^{2}-\omega^{2}} d x+\mu_{c t e}^{\prime}
$$

Where $\mu_{c t e}^{\prime}$ is a value that ensures the real permeability of the equivalent layer is 1 in low frequency.

\section{EXPERIMENTAL VALIDATION}

To test the method, we selected different inductive coils from WÜRTH Elektronik that cover multiple layer and one layers configurations and litz-wire and round conductors. The Finite Element models of these coils are simulated in Ansys Maxwell ${ }^{\circledR}$ where the actual winding is replaced by an equivalent layer with the magnetic and electrical properties obtained in Section IV and summarized in Table I.

The time-consuming for each case can be shown in Table II using Intel ® i7-core.

The different cases are:

Inductive link A. This coil [24] utilized Litz-wire with 100 strands and the diameter of each strand is $0.08 \mathrm{~mm}$. The total bundle has a diameter of $1.21 \mathrm{~mm}$. The diameter of the coil is $50 \mathrm{~mm}$ and the height are $4.5 \mathrm{~mm}$. Neither $3 \mathrm{D}$ and $2 \mathrm{D}$ simulation of this coil with the actual conductor are feasible due to the fine meshing required (in Table II it is reflected as N/A). Using the equivalent layer with homogeneous current as can be seen in Fig. 7, where the model utilized in Ansys Maxwell@ is illustrated, using the complex permeability shown in Fig. 7a and the conductivity shown in Fig. 7b, 3D simulation takes 16 s for meshing and 2 s per frequency solved. In $2 \mathrm{D}$, the meshing time is reduced by a factor of 3 and the solving frequency by a factor of 6 . Regarding the accuracy, it can be seen in Fig 7c that both inductance and AC resistance agree with measurements. Both facts make this method very suitable for optimization of inductive coil.

Inductive link B. The winding of this coil [25] is made of a one-layer of round conductors where we have two conductors in parallel with a diameter of $0.45 \mathrm{~mm}$. The dimensions of this coil are $40 \mathrm{~mm} \times 40 \mathrm{~mm} \times 1.5 \mathrm{~mm}$. The direct 3D simulation with the real conductors was not possible, and 2D simulation will required some post-processing due to the lack symmetry. It is possible 3D simulation using the proposed method: replacing the actual conductors by the equivalent layer with homogeneous current, it can be seen in Fig. 8. The orange part represents the winding as an equivalent layer and the gray part is the magnetic core made of ferrite. Then, using the anisotropic complex permeability shown in Fig. 8a and the conductivity shown in Fig. $8 \mathrm{~b}$ to model the equivalent layer, it is obtained good results (it can be shown in Fig. 8c). 3D simulation took 45 seconds for meshing and 4 seconds per frequency solution.

Inductive link C. The winding [26] is made of Litz-wire conductors with 80 strands and the diameters of each strands is $0.08 \mathrm{~mm}$. The total bundle has a diameter of $1.1 \mathrm{~mm}$. The dimensions of the coil C are $88 \mathrm{~mm} \times 52 \mathrm{~mm} \times 3.5 \mathrm{~mm}$.To introduce this model in Ansys Maxwell, both windings are replaced by independent equivalent layers as can be seen in Fig. 9. Both equivalent layers are excited by an homogeneous current and insulating boundary is set. To model the actual conductors, the parameters shown in Fig. 9a and Fig. 9b are used. In this case, because the winding is a double $\mathrm{D}$, the setup to measure this coil is the following: the primary winding is measured, and the secondary winding is in open circuit. Since both coils are equal, only one of them is measured. As in previous, 3D and 2D direct simulations are not feasible. 3D simulation using the equivalent layer proposed in this work is performed. The equivalent layer requited $65 \mathrm{~s}$ for meshing and 8s per frequency, also in Fig. 9c, AC resistance and inductance were accurated predicted.

Inductive link D. The winding is made of multiple layers of round conductors [27] with a diameter of $0.16 \mathrm{~mm}$. This coil is axisymmetric. The radio of this coil is $6 \mathrm{~mm}$ and the height is $1.6 \mathrm{~mm}$. All the conductors are replaced by a compact equivalent layer as can be seen in Fig. 10. In this 


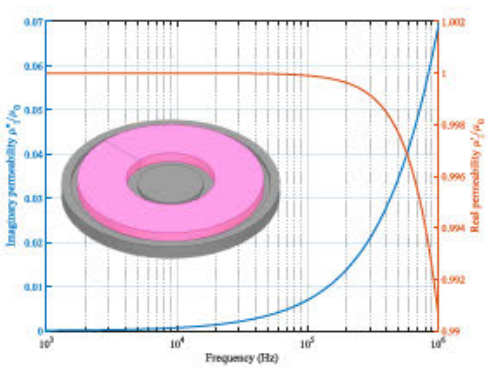

(a)

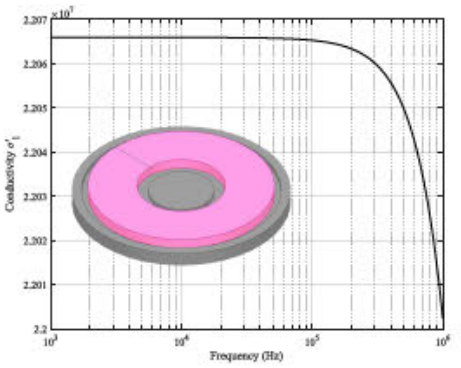

(b)

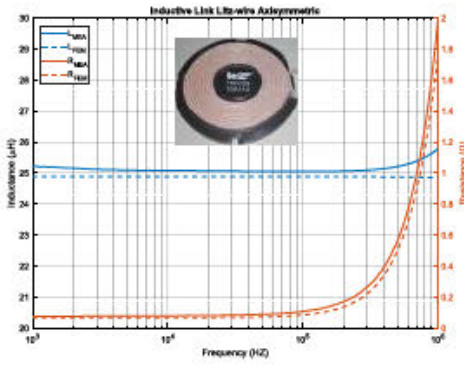

(c)

Figure 7: Equivalent layer parameters (a,b) and comparative results (c) of an axisymmetric inductive link with a winding made of Litz-wire.

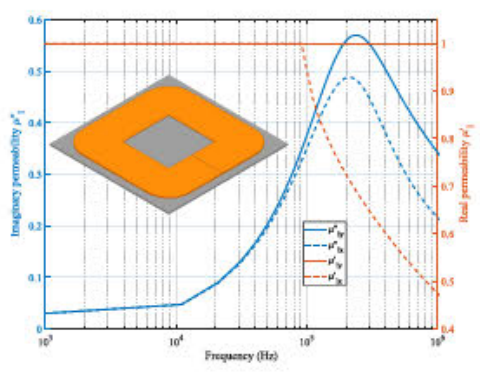

(a)

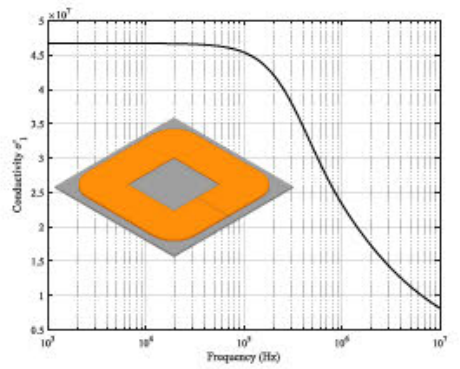

(b)

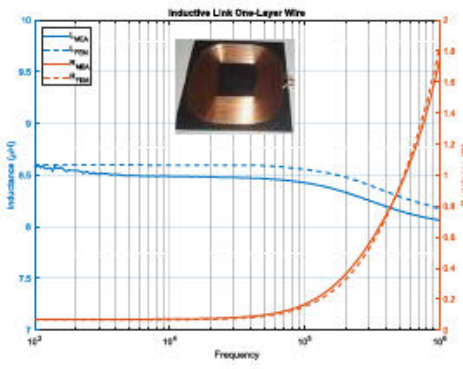

(c)

Figure 8: Equivalent layer parameters (a,b) and comparative results (c) of a rectangular coil with one-layer configuration winding.

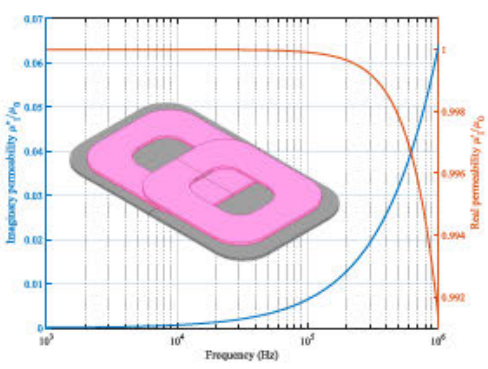

(a)

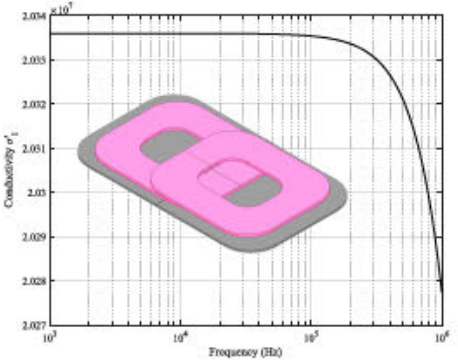

(b)

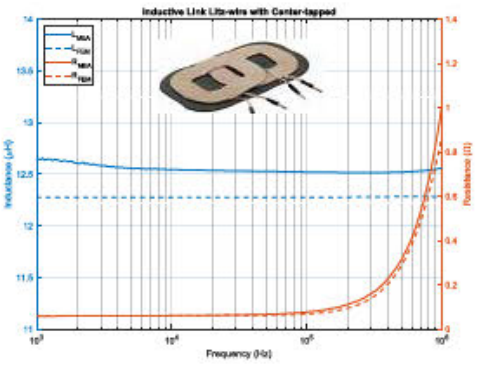

(c)

Figure 9: Equivalent layer parameters (a,b) and comparative results (c) of an inductive link with center-tapped made of Litz-wire.

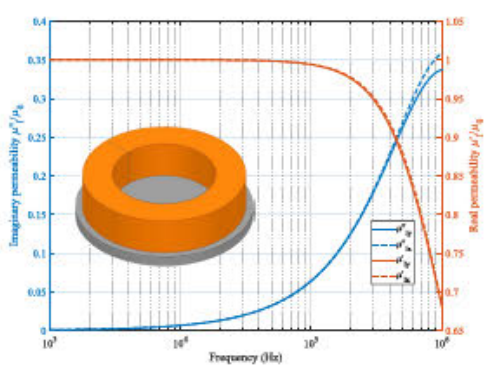

(a)

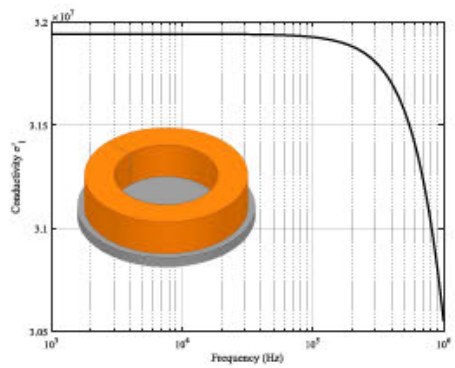

(b)

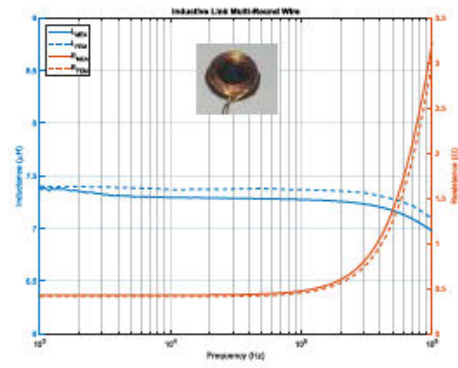

(c)

Figure 10: Equivalent layer parameters (a,b) and comparative results (c) of an axisymmetric coil with a winding made of round wire in Multi-round configuration. 


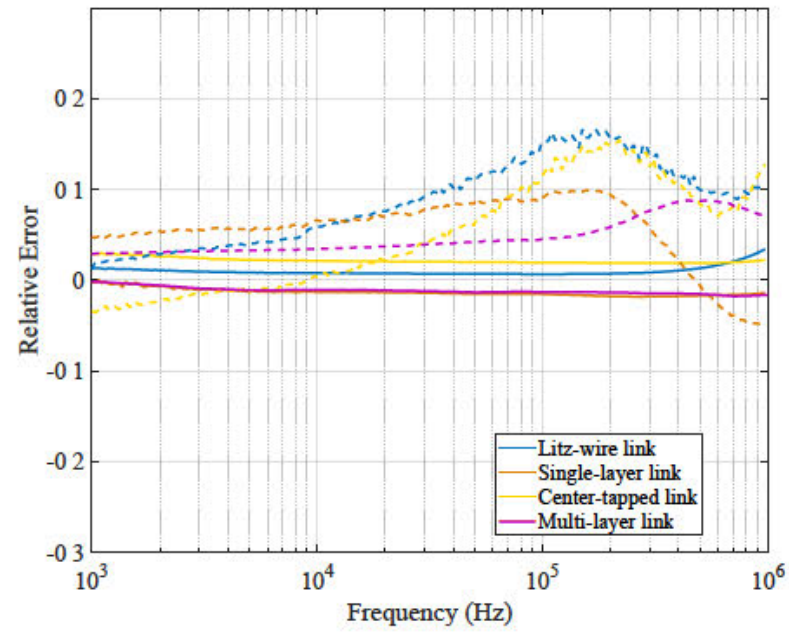

Figure 11: Relative error of the results shown in Fig. 7, Fig. 8, Fig. 9 and Fig. 10. The dashed lines represent the accuracy to predict the values of the inductances of the coils. Dashed lines represent the accuracy to predict the AC resistances value of the coils.

case, the complex permeability is anisotropic and it is shown in Fig. 10a; the conductivity is calculated as in the other cases and it can be seen in Fig. 10b. Again, 3D direct simulations were not possible due to the diameter, but in this case a $2 \mathrm{D}$ direct simulation was possible due to the symmetry. 2D and 3D simulation with the equivalent layer provided accurate results as can be seen in Fig. 10. It is interesting to notice that 3D simulation using the proposed method were faster than 2D simulation using actual conductors ( $4 \mathrm{x}$ faster per frequency). In the case of 2D simulation using equivalent layer, the simulation time is reduced by a factor of 60 .

From Fig. 11, it is noticed that the inductances results show good agreement between the simulations and the measurement. On the other hand, the $\mathrm{AC}$ resistance results present good agreement in low frequency, but about $100 \mathrm{kHz}$, that the $\mathrm{AC}$ resistance begins to grow, the model presents some discrepancies that can reach up to $15 \%$ error. After this point, the accuracy increase again as at low frequency.

\section{Conclusion}

In this work, a method to allow and to accelerate Finite Element Analysis of multiple layers and one-layer windings of round conductor and Litz-wire conductors is proposed. The method is based on the use of an equivalent layer with equivalent parameters that keeps macroscopic energy and losses of the multiple layers winding. The physical properties of the equivalent layer are obtained by means of analytical and empirical equations. The proposed method allows 3D Finite Element simulations that cannot be performed using detailed 3D Finite Element simulations with actual conductors. Additionally, it shows that even for simpler 2D simulations where detailed simulations with conductors are feasible, the proposed approach reduces the meshing time by a factor of 10 , and the solving time by a factor of 60 . This fact makes

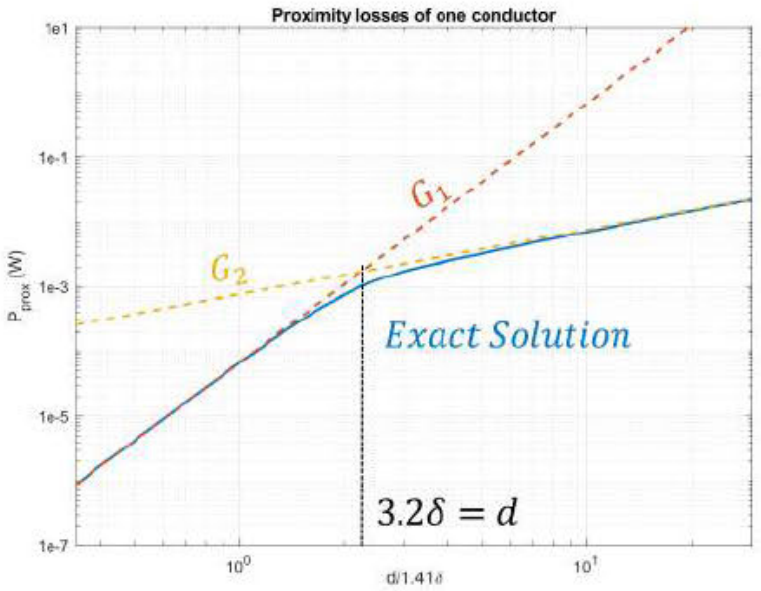

Figure 12: Proximity losses of one conductor of $1 \mathrm{~mm}$ of diameter under the influence of a magnetic field of $100 \mathrm{~A} / \mathrm{m}$. The orange line corresponds to function (27), which can predict low frequency losses. The yellow line corresponds to function (28), which can predict high frequency losses. The blue line is the exact solution of the losses of a conductor that corresponds to the simulation by finite elements.

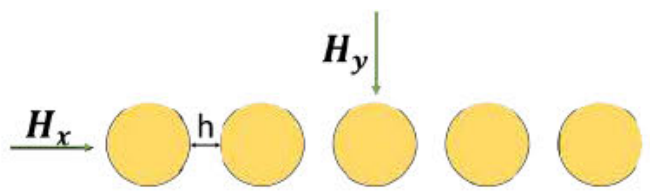

Figure 13: Finite Element Simulation configuration of OneLayer winding separated by an horizontal distance $h$.

this approach very appropriate for applying finite element simulation in the optimization of inductive power transfer links.

\section{APPENDIX A}

PROXIMITY LOSSES FUNCTION FOR SINGLE CONDUCTOR LAYER

Assuming a single conductor layer distributed in $\mathrm{x}$ direction, it is shown in Fig. 13, the proximity losses generated by an external magnetic field will be different is the field is in $\mathrm{x}$ and y direction. Then, two empirical expression for $G_{p}$ will be obtain both $\mathrm{x}$ and $\mathrm{y}$ direction.

In order to obtain these empirical expressions, two different set of finite element simulation have been performed with Ansys Maxwell@ with uniform magnetic field in $\mathrm{x}$ and $\mathrm{y}$ direction, independently.

The conductors have been separated by distance $h$ (varying from 0 to $\mathrm{d}$ ), the net current through the conductors has been set to $0 \mathrm{~A}$, the diameter $\mathrm{d}$ has been set in $1 \mathrm{~mm}$ and the frequency has been varied up to $10 \delta=d$. In order to simplify the analysis, symmetrical boundaries are used. It is described in the Fig. 14.

The first set of simulations are performed with uniform magnetic field in $\mathrm{x}$-direction while sweeping the distance and frequency as can be seen in Fig. 15a. The second set of 


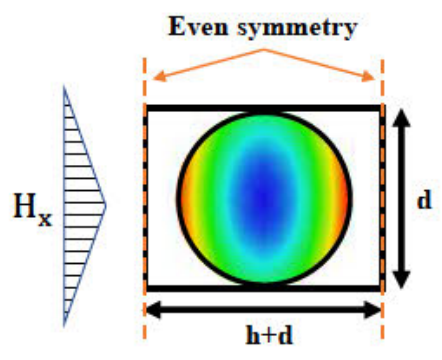

(a)

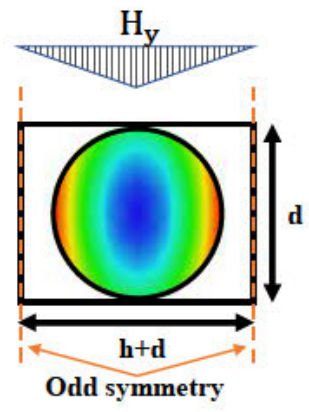

(b)
Figure 14: Illustration of the boundaries set in FE simulation to obtain the fitted model.

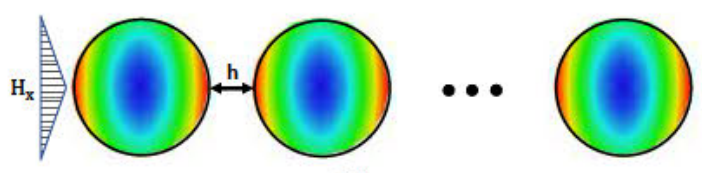

(a)

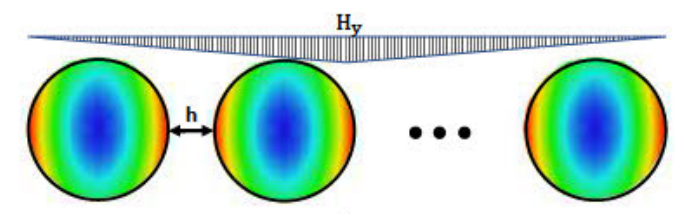

(b)

Figure 15: Illustration of the decoupled FE simulation to obtain the fitted model.

simulations are configured with uniform magnetic field in ydirection and sweeping the distance and the frequency. This second setup is shown in Fig. 15b.

From these simulations, we obtain the ohmic losses $\left(P_{F E M}\right)$ and the energy $\left(E_{F E M}\right)$ of the total layer. Then, the losses and the energy are normalized to the number of turns and fitted.

\section{A. Finite Element Fitting of $G_{p}$}

Using (2), we can obtain two empirical expression for $G_{p}$ as:

$$
G_{p_{x}}(\gamma)=\frac{\sigma P_{F E M_{x}}(\gamma)}{N_{t u r n s} H_{x}^{2}}
$$

And:

$$
G_{p_{y}}(\gamma)=\frac{\sigma P_{F E M_{y}}(\gamma)}{N_{t u r n s} H_{y}^{2}}
$$

Where $P_{F E M_{x}}$ and $P_{F E M_{y}}$ are the ohmic losses of the layer for the magnetic field in $\mathrm{x}$ and $\mathrm{y}$ direction respectively.

If we have one conductor which is excited with a certain magnetic field, we can predict the losses in low frequencies with (27) and in high frequency using (28): (as an example see Fig. 12)

$$
\begin{aligned}
G_{1}(\gamma) & =\frac{\pi}{8} \gamma^{4} \\
G_{2}(\gamma) & =\frac{2 \pi}{\sqrt{2}} \gamma
\end{aligned}
$$

To obtain a function that can predict exact losses and depends on (27) and (28), a weight function is needed to solve the problems at the turning point $3.2 \delta=d$ as:

$$
G_{p}(\gamma)=\frac{G_{1}(\gamma)}{f(\gamma)+1}+\frac{G_{2}(\gamma)}{f(\gamma)-1}
$$

Where

$$
\begin{aligned}
f(\gamma)= & \frac{0.08889 \gamma^{5}+0.03981 \gamma^{4}-0.1214 \gamma^{3}}{\gamma^{2}-0.4336 \gamma+0.06334} \\
& +\frac{0.9557 \gamma^{2}-2.174 \gamma^{1}+10.27}{\gamma^{2}-0.4336 \gamma+0.06334}
\end{aligned}
$$

Thus, the empirical expression for $G_{p x}$ and $G_{p y}$ when we have more turns can be expressed as:

$$
\begin{aligned}
& G_{p_{x}}\left(\gamma, N_{\text {turns }}, \frac{h}{d}\right)= \\
& \quad x\left(\frac{h}{d}, N_{\text {turns }}\right) \frac{G_{1}(\gamma)}{f(\gamma)+1}+\frac{G_{2}(d, \gamma)}{f(\gamma)-1}
\end{aligned}
$$

And:

$$
\begin{aligned}
& G_{p_{y}}\left(\gamma, N_{\text {turns }}, \frac{h}{d}\right)= \\
& \quad y\left(\frac{h}{d}, N_{\text {turns }}\right) \frac{G_{1}(\gamma)}{f(\gamma)+1}+\frac{G_{2}(\gamma)}{f(\gamma)-1}
\end{aligned}
$$

Where:

$$
\begin{aligned}
& x_{0}\left(N_{\text {turns }}\right)=\tanh \left(\left(N_{\text {turns }}-1\right) 0.3801\right) 0.36+0.7949 \\
& x_{1}\left(N_{\text {turns }}\right)=\tanh \left(\left(N_{\text {turns }}-1\right) 0.7288\right) 0.368+0.00197
\end{aligned}
$$

$x_{2}\left(N_{\text {turns }}\right)=\tanh \left(\left(N_{\text {turns }}-1\right) 0.4347\right)(-0.4869)+0.9984$

$x\left(\frac{h}{d}, N_{\text {turns }}\right)=\tanh \left(\frac{h}{d} x_{0}\left(N_{\text {turns }}\right)\right) x_{1}\left(N_{\text {turns }}\right)+x_{2}\left(N_{\text {turns }}\right)$

And:

$y_{0}\left(N_{\text {turns }}\right)=\tanh \left(N_{\text {turns }}-3.313\right)(-5.424)-10.09$

$$
y_{1}\left(N_{\text {turns }}\right)=\tanh \left(N_{\text {turns }}-3.219\right) 0.5631+2.147
$$

$$
y\left(h, N_{\text {turns }}\right)=y_{0}\left(N_{\text {turns }}\right)\left(\frac{h}{d}\right)^{0.35}+y_{1}\left(N_{\text {turns }}\right)
$$

\section{B. Finite Element Fitting of $\mu_{l}$}

In this case, instead of calculating the real part of the permeability of the layer using Kramers-Kronig ratio as in the cases of litz wire and multiple layer round conductors, it is possible to obtain an empirical expression of the real part of the complex permeability using (5) as:

$$
\mu_{l_{x}}^{\prime}=\frac{4 E_{F E M_{x}}}{\mu_{0} H^{2} A}
$$


And:

$$
\mu_{l_{y}}^{\prime}=\frac{4 E_{F E M_{y}}}{\mu_{0} H^{2} A}
$$

From the simulations results, it is possible to obtain an expression for the real part of the permeability that describes the energy for a magnetic field in $\mathrm{x}$ direction as:

$$
\begin{aligned}
& \mu_{r_{x}}^{\prime}\left(N_{\text {turns }}, \frac{h}{d}, \gamma\right)= \\
& 1-b\left(N_{\text {turns }}, \frac{h}{d}\right) \tanh \left(\sqrt{\gamma-1.5} a\left(N_{\text {turns }}, \frac{h}{d}\right)\right)
\end{aligned}
$$

Where $a$ and $b$ can be expressed as:

$$
\begin{aligned}
a\left(N_{\text {turns }}, h\right)= & 0.4+0.04215 N_{\text {turns }}-0.005358 \frac{h}{d} \\
& -0.002983 N_{\text {turns }}^{2}-0.006102 N_{\text {turns }} \frac{h}{d}
\end{aligned}
$$

$$
\begin{aligned}
b\left(N_{\text {turns }}, h\right)= & 0.6223+0.04548 N_{\text {turns }}-0.07698 \frac{h}{d} \\
& -0.002895 N_{\text {turns }}^{2}-0.01695 N_{\text {turns }} \frac{h}{d}
\end{aligned}
$$

For the field in the y-direction, we can simplify using a real permeability of 1 .

\section{ACKNOWLEDGMENT}

The authors would like to thank the Ministerio de Economia, Industria y Competitividad de España (under Grants DPI201680953-R and RTC-2016-4820-4) for their funding.

\section{REFERENCES}

[1] J. Ferreira, "Analytical computation of ac resistance of round and rectangular litz wire windings," in IEE Proceedings B (Electric Power Applications), vol. 139, pp. 21-25, IET, 1992.

[2] J. A. Ferreira, "Improved analytical modeling of conductive losses in magnetic components," IEEE transactions on Power Electronics, vol. 9, no. 1, pp. 127-131, 1994.

[3] E. Bennett and S. C. Larson, "Effective resistance to alternating currents of multilayer windings," Electrical Engineering, vol. 59, no. 12, pp. 1010-1016, 1940.

[4] W. G. Hurley, E. Gath, and J. G. Breslin, "Optimizing the ac resistance of multilayer transformer windings with arbitrary current waveforms," in 30th Annual IEEE Power Electronics Specialists Conference. Record.(Cat. No. 99CH36321), vol. 1, pp. 580-585, IEEE, 1999.

[5] P. Dowell, "Effects of eddy currents in transformer windings," in Proceedings of the Institution of Electrical Engineers, vol. 113, pp. 13871394, IET, 1966.

[6] W. G. Hurley and W. H. Wölfle, Transformers and inductors for power electronics: Theory, design and applications. John Wiley \& Sons, 2013.

[7] P. Wallmeier, "Improved analytical modeling of conductive losses in gapped high-frequency inductors," IEEE Transactions on Industry Applications, vol. 37, no. 4, pp. 1045-1054, 2001.

[8] F. Tourkhani and P. Viarouge, "Accurate analytical model of winding losses in round litz wire windings," IEEE Transactions on magnetics, vol. 37, no. 1, pp. 538-543, 2001.

[9] R. Wojda and M. Kazimierczuk, "Winding resistance of litz-wire and multi-strand inductors," IET Power Electronics, vol. 5, no. 2, pp. 257$268,2012$.

[10] R. P. Wojda and M. K. Kazimierczuk, "Winding resistance and power loss of inductors with litz and solid-round wires," IEEE Transactions on Industry Applications, vol. 54, no. 4, pp. 3548-3557, 2018.

[11] J. Mühlethaler, Modeling and multi-objective optimization of inductive power components. PhD thesis, ETH Zurich, 2012.

[12] R. Bosshard, Multi-objective optimization of inductive power transfer systems for EV charging. PhD thesis, ETH Zurich, 2015.
[13] R. Bosshard, J. W. Kolar, J. Mühlethaler, I. Stevanović, B. Wunsch, and F. Canales, "Modeling and $\theta-\alpha$-pareto optimization of inductive power transfer coils for electric vehicles," IEEE Journal of Emerging and Selected Topics in Power Electronics, vol. 3, no. 1, pp. 50-64, 2015.

[14] J. Serrano, J. Acero, I. Lope, C. Carretero, and J. M. Burdío, "A flexible cooking zone composed of partially overlapped inductors," IEEE Transactions on Industrial Electronics, vol. 65, no. 10, pp. 7762-7771, 2018.

[15] X. Nan and C. R. Sullivan, "Simplified high-accuracy calculation of eddy-current loss in round-wire windings," in 2004 IEEE 35th Annual Power Electronics Specialists Conference (IEEE Cat. No. 04CH37551), vol. 2, pp. 873-879, IEEE, 2004.

[16] X. Nan and C. R. Sullivan, "A two-dimensional equivalent complex permeability model for round-wire windings," in 2005 IEEE 36th Power Electronics Specialists Conference, pp. 613-618, IEEE, 2005.

[17] X. Nan and C. R. Sullivan, "An equivalent complex permeability model for litz-wire windings," IEEE Transactions on Industry Applications, vol. 45, no. 2, pp. 854-860, 2009.

[18] E. L. Barrios, A. Ursua, L. Marroyo, and P. Sanchis, "Analytical winding loss calculation for high-frequency low-permeability inductors," in 2017 IEEE 18th Workshop on Control and Modeling for Power Electronics (COMPEL), pp. 1-7, IEEE, 2017.

[19] D. Meeker, "Continuum representation of wound coils via an equivalent foil approach," Available on 1-5-2015 at Http://www. femm. info/examples/prox/notes. pdf, 2006.

[20] A. Delgado, G. Salinas, J. A. Oliver, J. A. Cobos, J. Rodríguez, and S. Premo, "Equivalent parameters of round and litz wire conductors to obtain an equivalent layer to accelerate finite element simulations of wireless power transfer system," in 2018 IEEE Energy Conversion Congress and Exposition (ECCE), pp. 7375-7379, IEEE, 2018.

[21] A. Delgado, G. Salinas, J. Rodríguez, J. A. Oliver, and J. A. Cobos, "Finite element modelling of litz wire conductors and compound magnetic materials based on magnetic nano-particles by means of equivalent homogeneous materials for wireless power transfer system," in 2018 IEEE 19th Workshop on Control and Modeling for Power Electronics (COMPEL), pp. 1-5, IEEE, 2018.

[22] H. A. Haus and J. R. Melcher, Electromagnetic fields and energy, vol. 107. Prentice Hall Englewood Cliffs, NJ, 1989.

[23] O. Moreau, L. Popiel, and J. L. Pages, "Proximity losses computation with a $2 \mathrm{~d}$ complex permeability modelling," IEEE Transactions on Magnetics, vol. 34, pp. 3616-3619, Sep. 1998.

[24] W. Elektronik, “We-wpcc-transmitter 760308100110." https://katalog. we-online.com/pbs/datasheet/760308100110.pdf, accessed 2019-07-25.

[25] W. Elektronik, "We-wpcc-receiver 760308102207." https://katalog. we-online.com/pbs/datasheet/760308102207.pdf, accessed 2019-07-25.

[26] W. Elektronik, "We-wpcc-array 760308104119." https://katalog. we-online.com/pbs/datasheet/760308104119.pdf, accessed 2019-07-25.

[27] W. Elektronik, "We-wpcc-receiver 760308101216." https://katalog. we-online.com/pbs/datasheet/760308101216.pdf, accessed 2019-07-25.

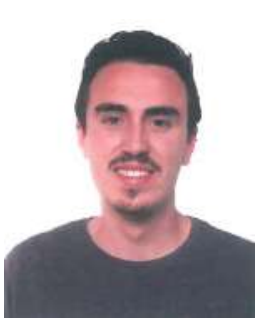

Alberto Delgado received the B.Sc. degree in Electrical Engineering at the University of Málaga (UMA), Spain, in 2016. During his undergraduate studies, he was awarded honors on several occasions and he achieved the Best Student of the Year Award. Furthermore, he received the M.Sc. degree in Industrial Electronics at the Universidad Politécnica de Madrid (UPM) in 2017. He became Teaching Assistant in 2019. He is currently working towards a Ph.D. degree in Industrial Electronics at UPM.

His research activities include modelling of dcdc converters for Inductive Power Transfer system, magnetic components for different applications such as RFID communications and wireless charging; and magnetic nano-materials and micro-materials. 


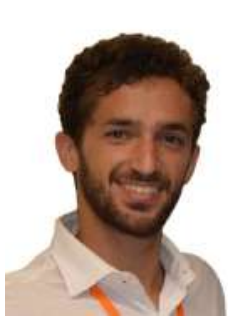

Guillermo Salinas received the B.Sc. degree in electronics engineering from the 'Universidad Miguel Hernández', Spain, and the M.Sc. degree in industrial electronics from the 'Universidad Politécnica de Madrid', Spain, in 2015 and 2016 respectively. During its stay in the Industrial Electronics Research Group in the UMH, he participate in the design and optimization of resonant power converters for space applications and contributed with his results to a research project about the analysis of the performance of GaN devices. The focus of his M.Sc. thesis, developed in the Industrial Electronics Center in the UPM, was in the development of a power distribution module for satellites, in collaboration with Thales-Alenia Space company.

His current $\mathrm{PhD}$ research interests are focused on the thermal modelling of high-frequency magnetic components. His models are developed for ANSYS, Thales-Alenia Space and SENER, among other companies.

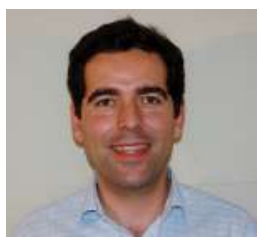

Jesús Ángel Oliver (M’00) received the Master and Doctoral degrees in Electrical Engineering from Universidad Politécnica de Madrid (UPM), Spain, in 1996 and 2007 respectively. He became Assistant Professor in 2001 and Associate Professor at UPM in 2007. He has been author and co-author in more than 150 scientific papers on Journals and Conferences and he holds 5 patents. His research activities include modelling (dc-dc converters, magnetic components, piezoelectric transformers, fuel-cells, and DC distributed power electronic systems), fast control techniques for dc-dc converters for VRM applications and RF amplifiers, three-phase rectifiers for aircraft applications, wireless power transfer and power systems on Chip.

Dr. Jesús A. Oliver has led numerous research projects with private and public funding and he has participated in more than 50 direct $\mathrm{R} D$ projects with companies in Europe, US, Australia and China. Currently he is serving as Associate Editor of the IEEE Transactions on Power Electronics.

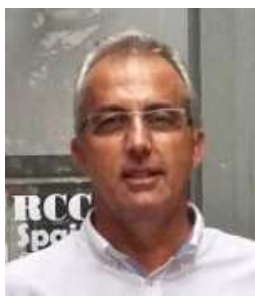

José Antonio Cobos (M'92-SM'12) is a Full Professor with the Universidad Politécnica de Madrid (UPM), Spain. In 2006, he was the Founder Director of the Centro de Electrónica Industrial at UPM (CEI-UPM), a university research center leading a strong industrial program in power electronics and digital systems. Since 2016, he has been the Founder President of its Industrial Council, to coordinate education and research with industry. His contributions are focused on power supply systems for industrial, aerospace, telecom, automotive, renewable energy, and medical applications. He advised over 50 graduate students, has published over 300 technical papers ( $>8000$ citations, $\mathrm{h}=47$ ), and is co-inventor of patents with 6 companies. His current research interests include energy efficiency in digital systems, RF amplifiers, renewable energy, magnetic components, transcutaneous energy transfer and biomedical applications. Dr. Cobos was an Adcom Member of the Power Electronics Society (IEEE-PELS) and the Chair of its Technical Committee on DC Power Supply systems. He was the General Chair of PwrSoC 2016 (IEEE-PELS and Power Supply Manufacturers Association, PSMA) and an Associate Editor of the IEEE Transactions on Power Electronics and the PELS Letters.

He conducted professional seminars and tutorials in USA, U.K., Austria, Germany, Italy, Sweden, Switzerland, Syria, Mexico, and Macedonia. He is a Steering Committee Member (Program Chair in 2019) of the IEEE Applied Power Electronics Conference (APEC). In 2016-17, he was an RCC Fellow at Harvard University, Cambridge, MA, USA and a Fulbrighter with the University of California at Berkeley, Berkeley, CA, USA.

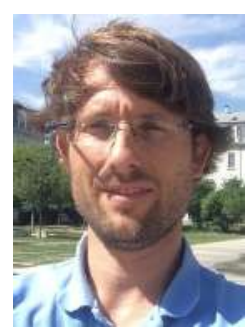

Jorge Rodriguez-Moreno Jorge Rodríguez-Moreno was born in Málaga, Spain in 1984. He received the B.S and M.S degree from Malaga University in Industrial engineering in 2010 and the $\mathrm{PhD}$. degree in Applied Physics in 2014. From 2015 has been working in the innovation center from Premo Group as engineer. During his $\mathrm{PhD}$. degree has been as visiting researcher in the Physics Institute of Freiburg, Germany and in the Lawrence Berkeley National Laboratory, USA during his Post-doctoral.

His research interest includes magnetic sensor, RFID components, materials characterization and supercapacitors. Has been awarded as co-author for one of his publication in the SUSChem award. 
